\title{
Angiotensin-II Modulates GABAergic Neurotransmission in the Mouse Substantia Nigra
}

\author{
Maibam R. Singh, Jozsef Vigh, and Gregory C. Amberg
}

https://doi.org/10.1523/ENEURO.0090-21.2021

Department of Biomedical Sciences, Colorado State University, Fort Collins, CO 80523

\begin{abstract}
GABAergic projections neurons of the substantia nigra reticulata (SNr), through an extensive network of dendritic arbors and axon collaterals, provide robust inhibitory input to neighboring dopaminergic neurons in the substantia nigra compacta (SNc). Angiotensin-II (Ang-II) receptor signaling increases SNc dopaminergic neuronal sensitivity to insult, thus rendering these cells susceptible to dysfunction and destruction. However, the mechanisms by which Ang-II regulates SNc dopaminergic neuronal activity are unclear. Given the complex relationship between SN dopaminergic and GABAergic neurons, we hypothesized that Ang-Il could regulate SNc dopaminergic neuronal activity directly and indirectly by modulating SNr GABAergic neurotransmission. Here, using transgenic mice, slice electrophysiology, and optogenetics, we provide evidence of an $\mathrm{AT}_{1}$ receptor-mediated signaling mechanism in SNr GABAergic neurons where Ang-Il suppresses electrically-evoked neuronal output by facilitating postsynaptic $G A B A_{A}$ receptors $\left(G A B A_{A} R s\right)$ and prolonging the action potential (AP) duration. Unexpectedly, Ang-II had no discernable effects on the electrical properties of SNc dopaminergic neurons. Also, and indicating a nonlinear relationship between electrical activity and neuronal output, following phasic photoactivation of SNr GABAergic neurons, Ang-II paradoxically enhanced the feedforward inhibitory input to SNc dopaminergic neurons. In sum, our observations describe an increasingly complex and heterogeneous response of the SN to Ang-II by revealing cell-specific responses and nonlinear effects on intranigral GABAergic neurotransmission. Our data further implicate the renin-angiotensin-system (RAS) as a functionally relevant neuromodulator in the substantia nigra, thus underscoring a need for additional inquiry.
\end{abstract}

Key words: angiotensin II; brain RAS; dopaminergic; GABAergic; neurotransmission; substantia nigra

\section{Significance Statement}

Angiotensin II (Ang-II) promotes dopamine release in the striatum and, in the substantia nigra compacta (SNc), exacerbates dopaminergic cell loss in animal models of Parkinson's disease (PD). Despite a potential association with PD, the effects of Ang-II on neuronal activity in the basal nuclei is unknown. Here, we describe a novel $A T_{1}$ receptor-dependent signaling mechanism in GABAergic projection neurons of the substantia nigra reticulata (SNr), a major inhibitory regulator of SNc dopaminergic neurons. Specifically, Ang-Il suppresses SNr GABAergic neuronal activity, subsequently altering GABAergic modulation of SNc dopaminergic neurons in a nonlinear fashion. Altogether, our data provide the first indication of Ang-II-dependent modulation of GABAergic neurotransmission in the SN, which could impact output from the basal nuclei in health and disease.

\section{Introduction}

Complementing the systemic cardiovascular renin-angiotensin-system (RAS), the CNS contains a fully-formed

Received March 9, 2021; accepted March 22, 2021; First published March 26, 2021.

The authors declare no competing financial interests. and independent RAS (Arnold et al., 2013; LabandeiraGarcía et al., 2014, 2017; Rocha et al., 2016; Valenzuela et al., 2016; Perez-Lloret et al., 2017; Jackson et al.,

Author contributions: M.R.S., J.V., and G.C.A. designed research; M.R.S. performed research; J.V. and G.C.A. contributed unpublished reagents/ analytic tools; M.R.S. and G.C.A. analyzed data; M.R.S., J.V., and G.C.A. wrote the paper. 
2018). Increasing evidence suggests that the peptide hormone angiotensin II (Ang-II), a primary RAS effector, contributes to neurodegenerative disorders such as Parkinson's disease (PD) and Alzheimer's disease (Arnold et al., 2013; Labandeira-García et al., 2014; Jackson et al., 2018). In animal models of PD, Ang-II-dependent activation of $\mathrm{AT}_{1}$ receptors promotes dopaminergic neuronal cell loss in the substantia nigra compacta (SNc; Grammatopoulos et al., 2007; Sonsalla et al., 2013).

Evidence also suggests that Ang-II evokes dopamine release in the rat striatum, and several studies report altered Ang-ll receptor expression levels in tissue samples from Parkinson's patients (Simonnet and Giorguieff-Chesselet, 1979; Allen et al., 1991; Brown et al., 1996; Ge and Barnes, 1996). Consistent with these observations, dopaminergic and GABAergic neurons in the $\mathrm{SN}$ of rodents and primates, including humans, express Ang-II receptors (Garrido-Gil et al., 2013). However, the occurrence of Ang-II-dependent modulation of GABAergic input to SNc dopaminergic neurons remains to be determined.

GABAergic projection neurons in the substantia nigra reticulata ( $\mathrm{SNr}$ ), located ventrolateral to the $\mathrm{SNc}$, through their axon collaterals, provides a major inhibitory input to SNc dopaminergic neurons (Parent, 1990; Mailly et al., 2003; Hikosaka, 2007; Tepper and Lee, 2007). Indeed, $>70 \%$ of the synapses onto SNc dopaminergic neurons are GABAergic (Smith and Bolam, 1990; Tepper and Lee, 2007). This intranigral inhibitory circuit regulates phasic dopaminergic output during behaviors such as reward extinction and conditioned negative association (Pan et al., 2013; Brown et al., 2014). Accordingly, inhibition of SNr GABAergic neurons causes disinhibition and burst firing of SNc dopamine neurons, leading to increased dopamine levels in the striatum and the basal nuclei (Tepper et al., 1995; Tepper and Lee, 2007; Lobb et al., 2011).

Regulation of $\mathrm{SNc}$ dopaminergic neurotransmission by $\mathrm{SNr}$ GABAergic neurons is well established and contributes to basal nuclei circuit dysfunction (Deniau et al., 2007; Rice et al., 2011). Prior investigations show that Ang-II, via activation of $\mathrm{AT}_{1}$ receptors, modulates GABAergic neurotransmission in the anterior hypothalamus, amygdaloid complex, and the median preoptic nucleus (Henry et al., 2009; Xing et al., 2009; Hu et al., 2018). Given the strong expression of Ang-II receptors throughout the $\mathrm{SN}$ and the predominantly GABAergic control of dopaminergic neurons, Ang-II signaling in SNr GABAergic neurons could potentially modulate intranigral GABAergic and dopaminergic neurotransmission. Despite the

This work was supported by the National Institutes of Health Grants 5R01HD087347 (to G.C.A.) and 5R01EY029227 (to J.V.).

Acknowledgements: We thank Dr. Shane Hentges, Dr. Andrew Rau, Dr. Susan Tsunoda, and Dr. Michael Tamkun for providing technical assistance, insightful critiques, and helpful feedback.

Correspondence should be addressed to Gregory C. Amberg at gregory. amberg@colostate.edu.

https://doi.org/10.1523/ENEURO.0090-21.2021

Copyright @ 2021 Singh et al.

This is an open-access article distributed under the terms of the Creative Commons Attribution 4.0 International license, which permits unrestricted use, distribution and reproduction in any medium provided that the original work is properly attributed. potential clinical relevance of Ang-II signaling in the SN, investigations of intranigral Ang-II functionality are lacking.

Here, we tested the hypothesis that Ang-II modulates SNr GABAergic neuronal activity, which, in turn, alters GABAergic regulation of SNc dopaminergic neurons. Using a combination of ex vivo brain slice electrophysiology and optogenetics, we find that Ang-II, via $A T_{1}$ receptors, acutely suppresses electrically evoked action potential (AP) firing of SNr GABAergic neurons. This effect is dependent on enhanced postsynaptic $\mathrm{GABA}_{A}$ receptor $\left(G A B A_{A} R\right)$ activity and prolonged AP duration (APD). Corresponding Ang-II-dependent effects were not evident in SNc dopaminergic neurons. However, on phasic photoactivation of SNr GABAergic neurons, Ang-ll paradoxically enhanced the feedforward inhibition of SNc dopaminergic neurons. This unexpected finding suggests that Ang-II's effect on postsynaptic SN dopaminergic neurons is nonlinear during synchronous GABAergic neuronal activity.

In sum, our data provide strong evidence of Ang-II signaling in SNr GABAergic neurons, disparate effects in $\mathrm{SNc}$ dopaminergic neurons, and illustrate the complex heterogeneity of the ensuing neuronal responses. Further, our observations reveal the intranigral microcircuitry as a potential target for modifying the basal nuclei output.

\section{Materials and Methods}

\section{Animals}

We bred the following two mouse strains from The Jackson Laboratory to generate a tdTomato reporter mice for dopaminergic neurons: Cre-dependent tdTomato reporter

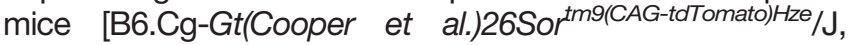
stock \#007909], and tyrosine hydroxylase (TH) promotordriven Cre expression (B6.Cg-7630403G23Rik ${ }^{T g(T h-c r e) 1 T m d} / \mathrm{J}$, stock \#008601). In order to focally stimulate SNr GABAergic neurons in ex vivo brain slices, we used transgenic mice expressing ChR2 fused to YFP under the control of mouse thymus cell antigen 1 (Thy 1) promoter (stock \#007612, The Jackson Laboratory), which specifically express ChR2 in SNr GABA neurons, but not in SN dopaminergic neurons. All mice used for the study were between four and eight weeks old. To detect transgene and floxed alleles, we used a genotyping service (Transnetyx).

\section{Animal care and euthanasia}

Mice received ad libitum access to standard chow and tap water while housed individually or in groups of less than four in a temperature and humidity-controlled room set on a 12/12 h light/dark cycle. On the day of experimentation, adult male and female mice were deeply anesthetized under isoflurane, decapitated, and the brains were removed and placed into an ice-cold artificial CSF (aCSF) containing the following: $126 \mathrm{~mm} \mathrm{NaCl}, 2.5 \mathrm{~mm}$ $\mathrm{KCl}, 1.2 \mathrm{~mm} \mathrm{MgCl}_{2}, 1.4 \mathrm{~mm} \mathrm{NaH}_{2} \mathrm{PO}_{4}, 25 \mathrm{~mm} \mathrm{NaHCO}_{3}, 11$ $\mathrm{mM}$ D-glucose, and $2.4 \mathrm{~mm} \mathrm{CaCl}_{2} ; \mathrm{pH} 7.4$, osmolarity $=310$ mOsm, bubbled with $95 \% \mathrm{O}_{2} / 5 \% \mathrm{CO}_{2}$. All procedures, including euthanasia, were performed according to institutional guidelines and approved by the Institutional Animal Care and Use Committee of Colorado State University. 


\section{Slice electrophysiology}

We used midbrain coronal slices to minimize the influence of presynaptic connections and carry out electrophysiological recordings in relative isolation. Coronal slices $(\approx 240 \mu \mathrm{m}$ thick) containing the SNr and SNc were cut (Leica VT1200S; Leica Microsystems), transferred to a holding chamber, and incubated for $30-60 \mathrm{~min}$ at $35^{\circ} \mathrm{C}$ in aCSF supplemented with MK-801 (100 $\mu \mathrm{M})$, and stored at $21^{\circ} \mathrm{C}$ until used for experimentation. To record from cells, we transferred individual slices to a chamber continuously perfused with $21-22^{\circ} \mathrm{C}$ aCSF at a rate of $2-3 \mathrm{ml} / \mathrm{min}$. We identified and differentiated nigral GABAergic and dopaminergic neurons based on their well-established electrophysiological features (described below) as well as expressed fluorescence in the respective transgenic lines: TdTomato for dopaminergic neurons (TH-Cre-TdTomato) and YFP for GABAergic neurons (Thy1-ChR2-YFP).

Borosilicate glass pipettes, with resistances of 3-5 M 2 , were fabricated on a laser micropipette puller (Model P-2000; Sutter Instrument). Whole-cell current-clamp recordings of evoked spike activity used a potassium gluconate-based intracellular solution composed of the following: $120 \mathrm{~mm} \mathrm{K-glu-}$ conate, $20 \mathrm{~mm} \mathrm{KCl}, 10 \mathrm{~mm}$ HEPES, $0.2 \mathrm{~mm}$ EGTA, $2 \mathrm{~mm}$ $\mathrm{MgCl}_{2}, 10 \mathrm{~mm}$ phosphocreatine, $2 \mathrm{~mm} \mathrm{Mg}$-ATP, and $0.3 \mathrm{~mm}$ Na-GTP; pH 7.5 adjusted with $\mathrm{KOH}$, osmolarity $=290-295$ mOsm). Pipettes for recording spontaneous postsynaptic currents (PSCs) and miniature IPSC (mIPSC) contained a high chloride potassium methylsulfate-based solution composed of the following: $57.5 \mathrm{~mm} \mathrm{~K}$-methyl sulfate, $57.5 \mathrm{~mm}$ $\mathrm{KCl}, 20 \mathrm{~mm} \mathrm{NaCl}, 1.5 \mathrm{~mm} \mathrm{MgCl}_{2}, 5 \mathrm{~mm}$ HEPES, $0.1 \mathrm{~mm}$ EGTA, $10 \mathrm{~mm}$ phosphocreatine, $2 \mathrm{~mm} \mathrm{Mg}$-ATP, and $0.3 \mathrm{~mm}$ Na-GTP; pH 7.5 adjusted with $\mathrm{KOH}$, osmolarity $=290-295$ mOsm.

We visualized cells with a $40 \times$ water immersion objective on an upright microscope (Zeiss) equipped with Dodt gradient contrast and collected data with an EPC-10 USB patch-clamp amplifier controlled with PatchMaster software (v.2.30; HEKA Electronik). Data were low-pass filtered at $10 \mathrm{kHz}$ and sampled at $50 \mathrm{kHz}$. We compensated for fast and slow capacitive transients, series resistance, and only used recordings with stable series resistances $<20 \mathrm{M} \Omega$. To ensure the fidelity of our measurements, we continuously monitored electrophysiological parameters, including series resistance, leak, and membrane voltage. Cells with unstable recording parameters, such as leak change of $>20 \%$ and high series resistance, were flagged and not used. GABAergic SNr neurons were electrophysiologically identified and distinguished from dopaminergic neurons by their well-characterized electrophysiological profile (Yung et al., 1991; Richards et al., 1997; Pan et al., 2013).

After gaining whole-cell access, cells were held at $-65 \mathrm{mV}$ (corrected for a liquid junction potential of $5 \mathrm{mV}$; Barry, 1994). Following stabilization, cells were hyperpolarized stepwise from -65 to $-140 \mathrm{mV}$ to measure $I_{h}$ and provide an initial characterization of the cell as either GABAergic or dopaminergic. Next, evoked APs were recorded in response to 2-s-long current injections, increasing stepwise from -50 to $150 \mathrm{pA}$, for at least $5 \mathrm{~min}$. After obtaining a series of stable recordings with aCSF (control), we superfused cells with aCSF supplemented with
Ang-II (500 nм) for a minimum of $5 \mathrm{~min}$ and recorded evoked APs for $\geq 15 \mathrm{~min}$. To conclude the experiment, Ang-Il was washed out by superfusion with standard aCSF for at least $5 \mathrm{~min}$, after which a series of final recordings were recorded. For experiments with the $A_{1}$ receptor antagonist losartan $(1 \mu \mathrm{M})$ and the $\mathrm{GABA}_{\mathrm{A}} \mathrm{R}$ antagonist picrotoxin $(1 \mu \mathrm{M})$, each drug was superfused alone or with Ang-II. For these recordings, we used the same experimental design as used for Ang-II alone.

We recorded spontaneous whole-cell outward PSC with a high chloride $(57.5 \mathrm{~mm})$ internal solution (described above) at a liquid junction potential-corrected setting of $-70 \mathrm{mV}$. We did not correct for leak and discarded any cell with leak $> \pm 100 \mathrm{pA}$ or a change in leak $> \pm 50 \mathrm{pA}$ during the length of the recording. Ang-II was either bath perfused for 3-5 min or puffed using a 1- to $3-\mathrm{M} \Omega$ glass pipette positioned ahead of the recording pipette and in the direction of laminar flow with Picospritzer III (Parker). To isolate and record spontaneous mIPSC at a holding potential of $-70 \mathrm{mV}$, we blocked excitatory synaptic transmission using a cocktail of drugs: the voltage-dependent sodium blocker tetrodotoxin (TTX; $500 \mathrm{nM}$ ), the

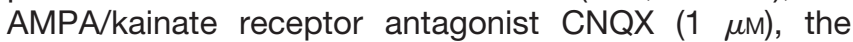
NMDA receptor antagonist MK-801 $(1 \mu \mathrm{M})$, and the nicotinic receptor antagonist hexamethonium bromide (100 $\mu \mathrm{M})$. IPSCs were confirmed to be mediated by $\mathrm{GABA}_{\mathrm{A}} \mathrm{Rs}$ with picrotoxin $(100 \mu \mathrm{M})$.

\section{Photostimulation and slice electrophysiology}

To generate light-evoked EPSP from ChR2 expressing SNr GABA neurons, we applied three 100-ms-long light pulses with an interstimulus interval of 2 s using a $470-n m$ LED (Thorlabs) driven by a LEDD1B driver (Thorlabs). Intrabusrt EPSP and spikes from three light-evoked responses were averaged with a minimum of three to five recordings from each group. We used a K-gluconate based internal solution (described above) for recording light-evoked EPSP and IPSC. IPSCs in SNc dopaminergic neurons were recorded in the presence of excitatory synaptic blockers and identified as outward current deflections in response to photostimulation of SNr GABA neurons. For analysis, we included only SNc dopaminergic neurons with outward current deflections in response to photostimulation of SNr GABAergic neurons. Similarly, neurons with light-evoked EPSPs were classified as GABAergic, whereas neurons showing light-evoked IPSP were classified as dopaminergic. EPSP and IPSP were recorded in current-clamp mode at $-65 \mathrm{mV}$ (adjusted for junction potential) using the K-gluconate based internal solution.

\section{Drugs}

We purchased CNQX, MK-801, Ang-II, losartan, and picrotoxin from Sigma and TTX and PD123319 from Tocris Biosciences. Drugs were prepared immediately before use in either distilled water (Ang-II, TTX, PD123319, and losartan) or DMSO (CNQX, MK-801, and picrotoxin) and diluted in aCSF to achieve the desired concentration. We used 500 nм Ang-II for our experiments, a high 
A

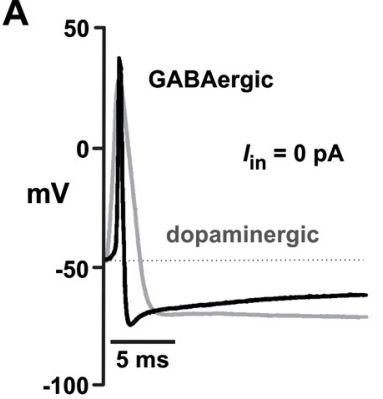

C

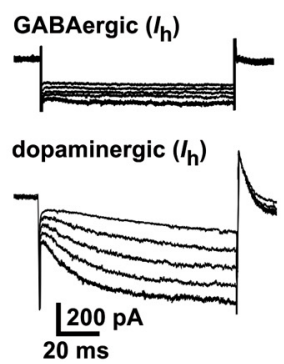

B

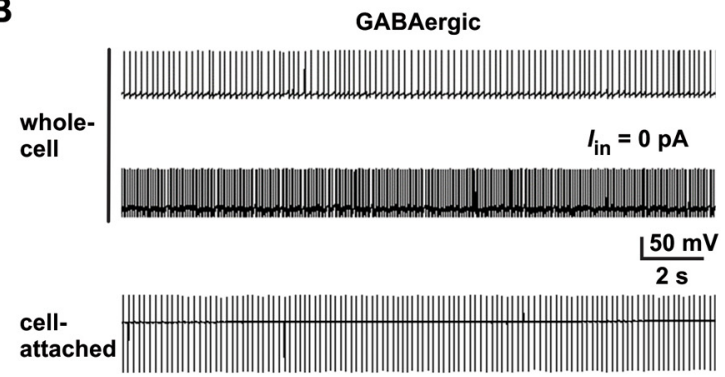

D



Figure 1. Electrophysiological characteristics of GABAergic and dopaminergic neurons in the mouse substantia nigra. $\boldsymbol{A}$, SNr GABAergic neurons have a narrower $(<1.5 \mathrm{~ms})$ AP width than SNc dopaminergic neurons $(>3 \mathrm{~ms})$. B , Representative sustained high-frequency firing of SNr GABAergic neurons recorded in whole-cell (top) and cell-attached configuration (bottom). $\boldsymbol{C}$, SNr GABAergic neurons, in contrast to SNc dopaminergic neurons, show little to no $I_{\mathrm{h}}$ in response to a series of hyperpolarizing pre pulses (-70 to $-140 \mathrm{mV}$ ). $\boldsymbol{D}$, Representative low-frequency, slow, and irregular pacemaker-like firing in SNc dopaminergic neurons.

working concentration for a ligand-receptor pair with a $K_{d}$ $<5 \mathrm{~nm}$. However, we used a high Ang-Il concentration to ensure adequate slice penetration of bath-applied Ang-II.

\section{Experimental design and statistical analyses}

Data were analyzed using Axograph X and GraphPad Prism (v.8) software. We used one-way, two-way ANOVA, mixed-effect analyses, and paired Student's $t$ test as indicated. Individual points in the figures represent data from a single cell. We performed only one experiment per brain slice and obtained no more than three recordings per mouse. We used male and female mice for all experiments. However, a sex-difference analysis revealed nothing of significance; thus, we pooled our data. Averaged data are presented as the mean \pm SEM unless otherwise specified; $n=$ number of cells recorded from, and significance was defined as $p<0.05$ unless otherwise indicated.

\section{Results}

To test our hypothesis that Ang-II regulates the activity of GABAergic projection neurons in the mouse SNr, we formulated the following requisite experimental criteria: (1) exogenous Ang-II must alter the AP firing characteristics of positively identified SNr GABAergic neurons in ex vivo brain slices; (2) Ang-II must promote changes in the electrophysiological properties of SNr GABAergic neurons by mechanisms consistent with the observed changes in AP firing behavior; (3) the observed effects of Ang-II must be sensitive to pharmacological blockade of cognate Ang-II receptors; (4) Ang-II should suppress light-evoked EPSP in ChR2 expressing SNr GABAergic neurons; and (5) Ang-
II must modulate GABAergic input onto postsynaptic SNc dopaminergic neurons.

\section{Ang-II suppresses evoked APs in SNr GABAergic neurons}

We performed ex vivo whole-cell electrophysiology on freshly prepared coronal midbrain slices from TdTomato dopaminergic neuron reporter mouse to investigate the effects of Ang-II on GABAergic projection neurons in the SNr. To begin, we used whole-cell current-clamp to record spontaneous and depolarization-evoked APs. As a means to provisionally identify and distinguish GABAergic neurons from dopaminergic neurons, we selected cells by their lack of tdTomato fluorescence and neuroanatomical features (see Materials and Methods). After obtaining electrophysiological access, we confirmed or contested cell identities using the well-characterized electrophysiological profiles of GABAergic and dopaminergic neurons (Lacey et al., 1989; Tepper et al., 1995; Richards et al., 1997). Thus, criteria used to categorize cells as GABAergic included: (1) an apparent absence of tdTomato fluorescence; (2) the presence of sustained high-frequency spontaneous AP firing $(>10 \mathrm{~Hz})$; (3) an AP width of $<2 \mathrm{~ms}$; (4) little or no hyperpolarization currents; and (5) minimal adaptation to injected depolarizing currents (Fig. $1 A-C$ ). Alternatively, we identified nigral dopaminergic neurons based on their cell size (25-40 M $)$ ), detectable tdTomato fluorescence, slow pacemaker-like AP firing, APDs $>2 \mathrm{~ms}$, robust hyperpolarization currents, and pronounced adaptation to injected depolarizing currents.

To achieve a relatively controlled measure of AP activity, we used an evoked AP protocol consisting of 2-s current injections increasing incrementally from -50 to 

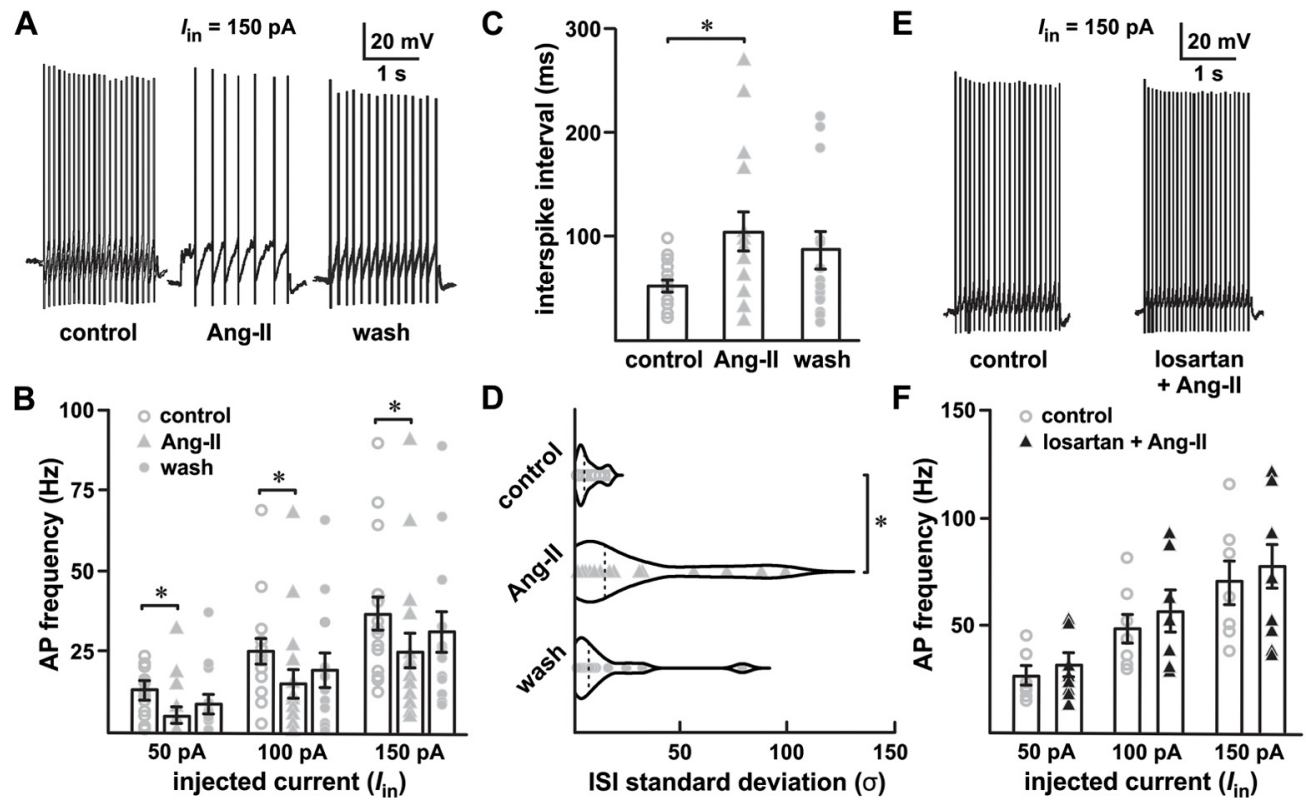

Figure 2. Ang-II decreases SNr GABAergic projection neuron spike firing. $\boldsymbol{A}$, Evoked APs in response to 150-pA current injection. $\boldsymbol{B}$, Individual scatter plot of evoked spike frequency in the SNr GABAergic projection neurons in response to 50-, 100-, and 150-pA current injections. Ang-II significantly decreased evoked firing in all stimulus levels combined, $50-150 \mathrm{pA}\left({ }^{\star \star \star} p<0.001\right.$, two-way repeated-measures ANOVA). In 10 out of 17 cells, washout partially reversed the effect of Ang-II. C, Ang-II increased the SNr GABAergic interspike interval (ISI; ${ }^{* *} p=0.005$, mixed effect analysis) and $(\boldsymbol{D})$ increased the irregularity of firing as quantified by the ISI SD ( ${ }^{*} p=0.01$, mixed effect analysis). $\boldsymbol{E}, \boldsymbol{F}$, The $\mathrm{AT}_{1}-\mathrm{R}$-specific blocker losartan $(1 \mu \mathrm{M})$ abolished the suppression of evoked $\mathrm{SNr}$ GABAergic spike firing by Ang-II.

$+150 \mathrm{pA}$. In cells identified as SNr GABAergic neurons obtained from a total of 13 mice, following control recordings in unsupplemented aCSF, bath-applied Ang-II (0.5 $\mu \mathrm{M})$ decreased evoked AP firing during current injections of 50,100 , and $150 \mathrm{pA}\left(n=17,{ }^{\star \star} p=0.003\right.$ for $50 \mathrm{pA}$, ${ }^{* \star \star} p<0.001$ for 100 and $150 \mathrm{pA}, F_{(2,32)}=9.521$; mixed-effect analysis; Fig. 2A,B). In 10 out of 17 cells, the effect of Ang-II on evoked AP firing was partially reversible with washout. Illustrating the degree of AP firing suppression, Ang-II increased the mean interspike interval by approximately 2-fold $\left(n=17, F_{(2,29)}=5.976, p=0.005\right.$; mixed-effect analysis; Fig. 2C). Additionally, Ang-II also increased the variability (i.e., irregularity) of the interspike intervals $(n=16$, $F_{(2,28)}=5.488, p=0.01$; mixed-effect analysis; Fig. 2D).

Indicative of $A T_{1}$ receptor involvement, preincubation with losartan $(1 \mu \mathrm{M})$ abolished decreased evoked AP firing following Ang-II application (Fig. 2E,F). To test for potential contributions by Ang-II type 2 receptors $\left(\mathrm{AT}_{2} \mathrm{Rs}\right)$, we used the specific $A T_{2} R$ antagonist PD123319. In contrast to $\mathrm{AT}_{1}$ receptor blockade with losartan, PD123319 (1 $\left.\mu \mathrm{M}\right)$ did not block Ang-II-dependent suppression of $\mathrm{SNr}$ GABAergic neuronal activity $\left(n=5, F_{(1,4)}=5.896\right.$, $p=0.072$, paired Student's $t$ test; data not shown). From these data, we conclude that Ang-II decreases evoked AP firing in SNr GABAergic neurons via $\mathrm{AT}_{1}$ receptor signaling. Note that we collected comparable data on identified SNc dopaminergic neurons (data not shown). However, the well-described adaptive responses of dopaminergic neurons to depolarization, and eventual depolarization block, precluded meaningful interpretation of these data (Lacey et al., 1989; Richards et al., 1997).

\section{Ang-Il prolongs APDs in SNr GABAergic neurons}

Sustained spontaneous high-frequency AP firing (10$15 \mathrm{~Hz}$, in vitro) is a striking characteristic of $\mathrm{SNr}$ GABAergic neurons (Lacey et al., 1989; Richards et al., 1997; Atherton and Bevan, 2005; Zhou and Lee, 2011). The high-frequency firing in these cells is autonomously generated and involves multiple ion channels from more than five families (Zhou et al., 2008; Seutin and Engel, 2010; Ding et al., 2011). We found that in SNr GABAergic neurons, Ang-II prolonged the duration of high-frequency APs in a largely reversible fashion (Figs. 3, 4). Indeed, Ang-II increased the half-width of both evoked $(n=17$; $F_{(2,29)}=5.440 ; p=0.010$; mixed-effect analysis; Fig. $\left.3 B\right)$ and spontaneous APs $\left(n=5 ; F_{(2,8)}=9.481 ; p=0.008\right.$; oneway repeated measures ANOVA; Fig. $4 C$ ). The effects of Ang-II on APD were prevented by preincubation with los$\operatorname{artan}(1 \mu \mathrm{M}$; Fig. 4B).

Further analysis of evoked APs revealed slower AP kinetics after Ang-II application in 11 out of $17 \mathrm{SNr}$ GABAergic neurons (same set of cells as in Fig. 2). Specifically, Ang-ll slowed evoked AP rise time $(n=9$; $F_{(2,16)}=7.683 ; p=0.005$; repeated measures one-way ANOVA; Fig. $3 C)$ and slowed AP decay $\left(n=10 ; F_{(2,18)}=\right.$ 7.102; $p=0.005$; repeated measure one-way ANOVA; Fig. $3 D$ ). Although no apparent effect on spontaneous AP rise times was evident, Ang-Il also slowed the decay of spontaneous APs in GABAergic neurons $\left(n=5 ; F_{(2,8)}=9.207\right.$; $p=0.015$; repeated measures one-way ANOVA; Fig. $4 C$ ). Interestingly, and contrasting SNr GABAergic neurons, cells identified as dopaminergic had no observable changes in APD or kinetics in response to Ang-II (Fig. 4D). These data 

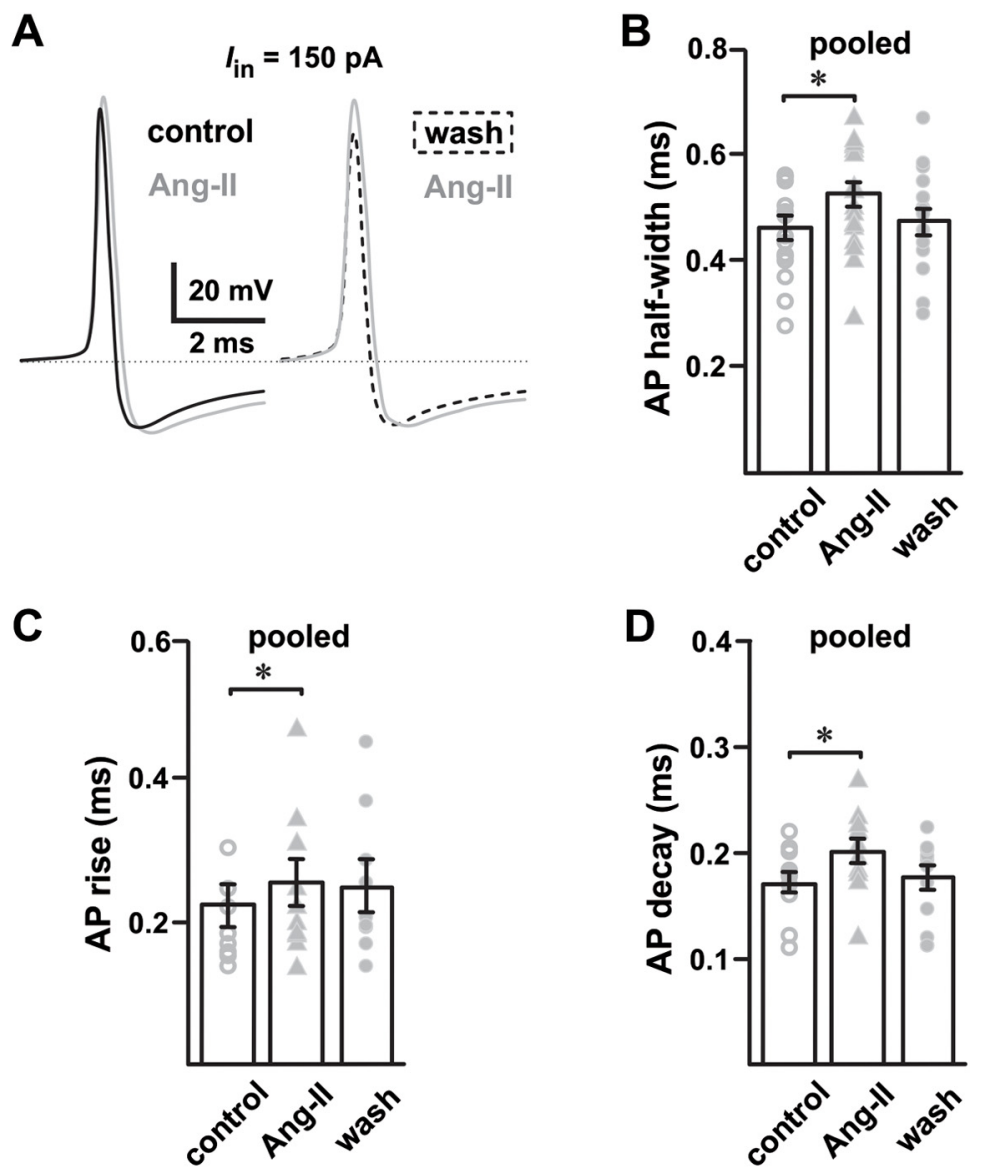

Figure 3. Ang-Il slows the evoked AP kinetics of SNr GABAergic neurons. A, Representative AP waveforms of SNr projection neurons showing that Ang-Il reversibly slows AP kinetics. $B$, Ang-II increased the AP half-width in 11 out of 17 SNr GABAergic neurons $\left({ }^{*} p=0.010\right.$, mixed-effect analysis). C , Ang-Il slowed the rise of APs in SNr GABAergic neurons $\left({ }^{* *} p=0.005\right.$, repeated measures oneway ANOVA). $\boldsymbol{D}$, Ang-Il slowed the decay of APs in SNr GABAergic neurons $\left({ }^{*} p=0.004\right.$, repeated measure one-way ANOVA). Pooled data are a grouped data of all stimulus levels $(50,100$, and $150 \mathrm{pA})$.

suggest that $A T_{1}$ receptor activation by Ang-Il prolongs APDs of SNr GABAergic but not SNc dopaminergic neurons. Further, the observed suppression of AP firing of $\mathrm{SNr}$ GABAergic neurons following Ang-II administration could arise, at least in part, as a consequence of AP prolongation because of a slowed rise and decay kinetics.

\section{Ang-II potentiates postsynaptic $\mathrm{GABA}_{\mathrm{A}} \mathrm{Rs}$ in $\mathrm{SNr}$ GABAergic neurons}

Striatal GABAergic medium-sized spiny neurons provide tonic inhibitory input to the SNr GABAergic neurons (Dray, 1979; McGeer et al., 1984; Smith and Bolam, 1990). These GABAergic neurons in the SNr express mostly $\mathrm{GABA}_{A}$ Rs but few $\mathrm{GABA}_{B}$ receptors. Indeed, electrical stimulation of the striatum produces short-term inhibition of SNr GABAergic neurons that is blocked by the $\mathrm{GABA}_{A} \mathrm{R}$ antagonists such as picrotoxin (Precht and Yoshida, 1971; Dray, 1979). To test whether the observed suppression of SNr GABAergic neuronal activity by Ang-II involves $\mathrm{GABA}_{A}$ Rs (Fig. 2), we replicated our evoked AP experiments in the presence of $G A B A_{A} R$ antagonist picrotoxin. Suggesting $\mathrm{GABA}_{A} R$ involvement, preincubation with picrotoxin $(1 \mu \mathrm{M})$ attenuated Ang-II-dependent suppression of
GABAergic neuronal firing (different set of four age matched TH-tdT mice; Fig. 5B). Interestingly, picrotoxin did not completely prevent Ang-II from prolonging the APD and slowing the AP rise time $\left(n=5, t_{(4)}=2.104, p=0.103\right.$; Student's paired $t$ test; Fig. $5 C)$, decay $\left(n=5, t_{(4)}=2.122, p=0.101\right.$; Student's paired $t$ test; Fig. $5 C)$, and half-width $\left(n=5, t_{(4)}=1.982\right.$, $p=0.119$; Student's paired $t$ test; Fig. $5 C$ ) in these cells. These data suggest that Ang-II potentially suppresses the excitability of SNr GABAergic neurons by two independent mechanisms (slowed AP kinetics and potentiation of $G A B A_{A} R s$ ).

To further examine the effects of Ang-II-dependent modulation of $\mathrm{GABA}_{A} \mathrm{R}$ activity in SNr GABAergic projection neurons, we recorded spontaneous PSCs (different set of eight age-matched TH-tdT mice; Fig. 6). Although Ang-Il did not significantly alter the frequency or average amplitude of spontaneous PSC in these cells (Fig. 6B), we did observe a roughly 2 -fold increased incidence of highamplitude spontaneous PSC, which are $3 \times$ the mean amplitude $\left(\geq-150 \mathrm{pA} ; n=9 ; t_{(8)}=3.243,{ }^{*} p=0.012\right.$; paired Student's $t$ test; Fig. 6B). Finally, we replicated our spontaneous PSC experiments in the presence of AP and excitatory synaptic blocker cocktail (see Materials and Methods) to confirm that the Ang-II-dependent increases in PSC are mediated postsynaptically. Synaptic blockade 
A

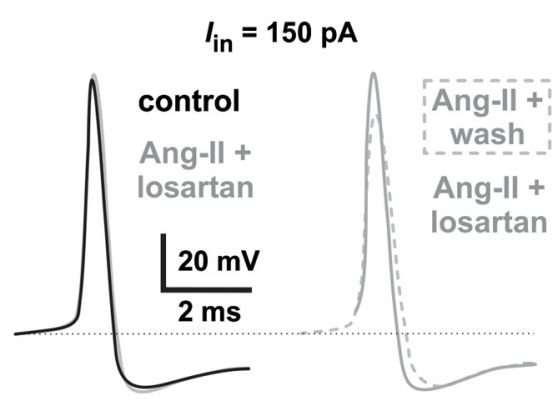

B

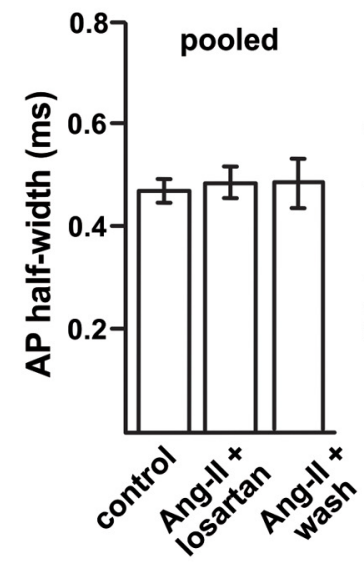

C

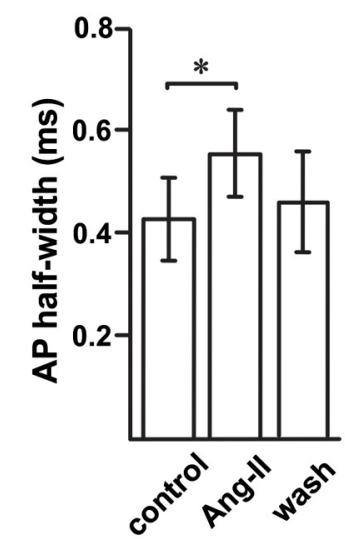

GABAergic spontaneous APs $\left(l_{\text {in }}=0 \mathrm{pA}\right)$
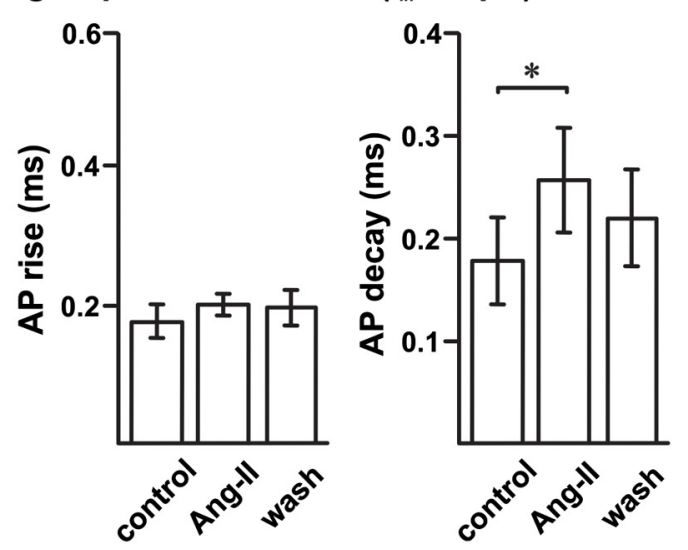

D
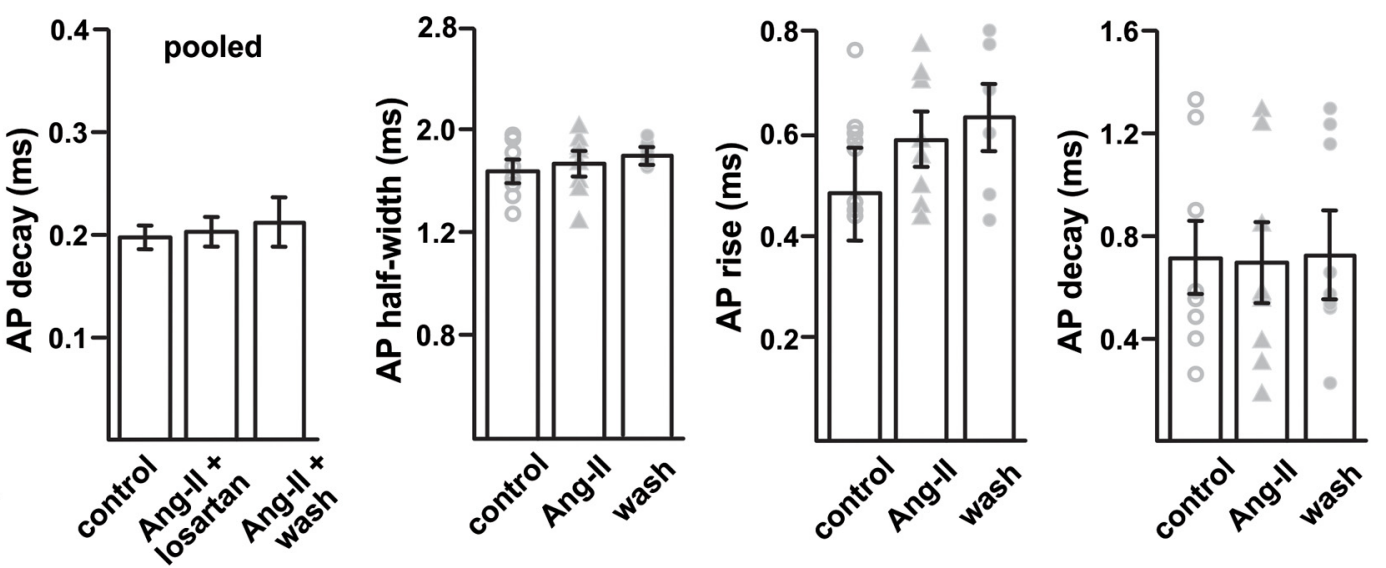

Figure 4. Disparate effect of Ang-II on SN GABAergic and dopaminergic neurons. A, Losartan blocks Ang-II-mediated increase in APD in SNr GABAergic neurons; losartan washout with continued Ang-Il perfusion prolonged the APD. B, Summary data showing that losartan $(1 \mu \mathrm{m})$ blocks Ang-Il-mediated increase in AP half-width and decay. Data from all stimulus levels (50, 100, and 150 pA) were pooled for each group. $\boldsymbol{C}$, Ang-II increased the duration of SNr GABAergic neuron spontaneous APs $\left({ }^{*} p=0.04\right.$, AP half-width; ${ }^{*} p=0.015$, AP decay; repeated measures one-way ANOVA). $\boldsymbol{D}$, Unlike SNr GABAergic neurons, Ang-II had no noticeable effect on the AP kinetics of SNc dopaminergic neurons.

drastically reduced the overall activity in our recordings to reveal the presence of spontaneous mIPSC (different set of seven age-matched TH-tdT mice; Fig. 7A). Similar to our experiments in the absence of synaptic blockers (Fig. 6), Ang-II significantly increased the observed incidence of high amplitude mIPSC ( $\geq 3 \times$ the mean amplitude; $\geq-121.86 \mathrm{pA}$ ), by $\sim 2.5$-fold without a significant change in the average mIPSC amplitude or frequency $\left(n=12 ; t_{(11)}=4.206,{ }^{\star \star} p=0.001\right.$; paired Student's $t$ test; Fig. 7A,B). Notably, picrotoxin (1 $\mu \mathrm{M})$ abolished the Ang-II-dependent increase in high-amplitude mIPSC in all cells (Fig. 7A). We repeated the experiment using a Picospritzer III (Parker) to provide a localized puff of Ang-II near the patched cell; however, we did not observe a noticeable change in high amplitude mIPSC (data not shown). In contrast, when Ang-II was reintroduced into the bath, the increase in high amplitude IPSC occurred as before (data not shown). This observation suggests that the $\mathrm{GABA}_{A} R$ s affected by Ang-II are likely located in distant dendritic terminals away from the soma and are embedded deep within the slice and cannot be reached with a topical puff of Ang-II. From these data, we conclude that Ang-II potentiates postsynaptic GABA ${ }_{A} R$ s in SNr GABAergic neurons, supporting our initial observation of suppressed excitability of nigral neurons by Ang-II (Fig. 2).

\section{$\mathrm{GABA}_{\mathrm{A}} \mathrm{Rs}$ in SNc dopaminergic neurons are not affected by Ang-II}

We also recorded spontaneous mIPSC from SNc dopaminergic neurons and found minimal background mIPSC compared with SNr GABAergic neurons (Fig. 7B,C). Further, Ang-II did not produce a detectable change in the spontaneous mIPSC of SNc dopamine neurons (Fig. 7C). This absence of effect suggests that in the basal state, in contrast to $\mathrm{SNr}$ GABAergic neurons, Ang-II has very little or no direct postsynaptic effect on the GABAergic activity in SNc dopaminergic neurons.

\section{Ang-II increases GABAergic input to SNc dopaminergic neurons}

In our previous experiments, Ang-II suppressed electrically evoked spike output in individual GABAergic 

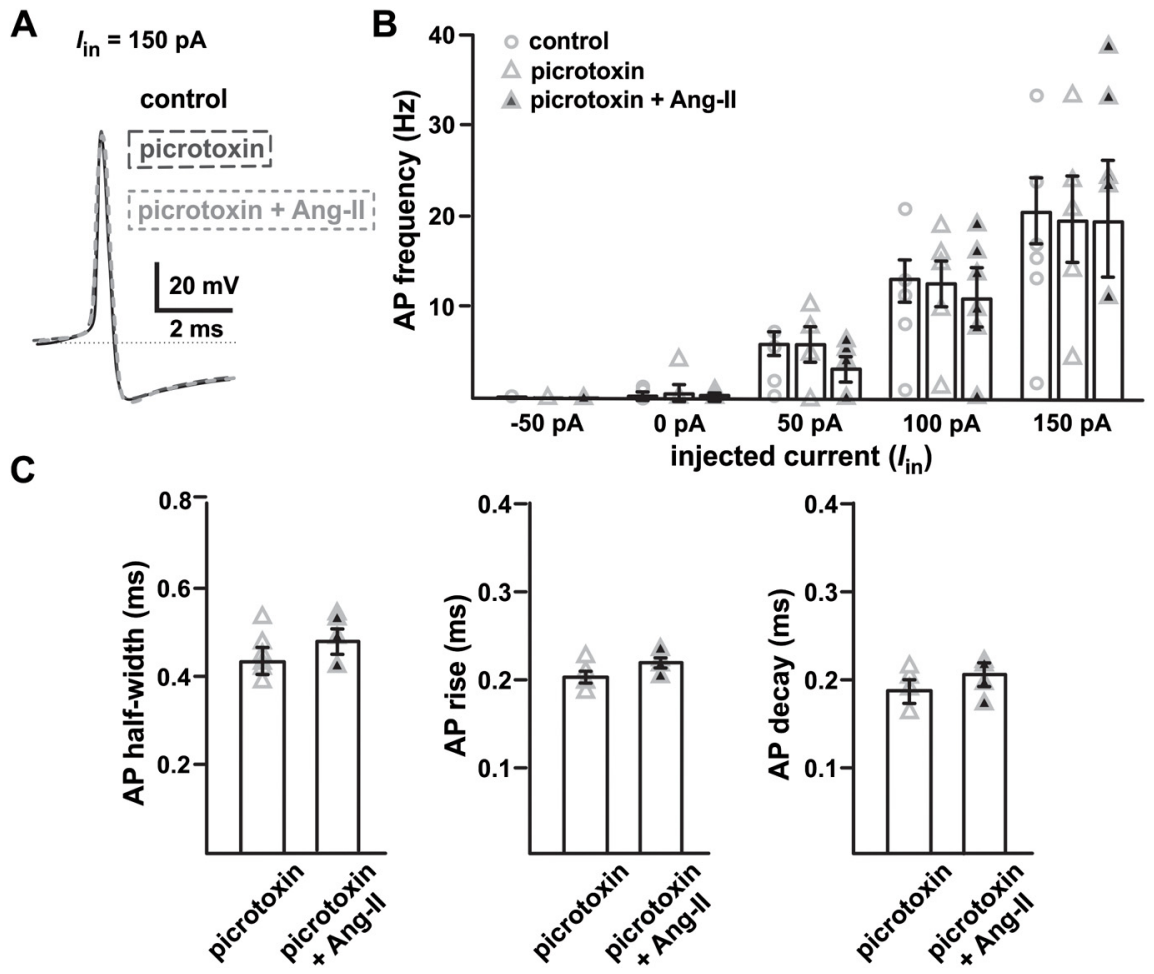

Figure 5. $\mathrm{GABA}_{\mathrm{A}} \mathrm{R}$ blockade prevents Ang-II-mediated suppression of evoked spike firing in the SNr GABAergic projection neurons. $A$, Overlaid spike waveforms for control conditions, in the presence of the GABA $\mathrm{R}$ antagonist picrotoxin (1 $\mu \mathrm{M})$, and in the presence of picrotoxin plus Ang-II. B, Summary plot showing evoked spike firing of SNr GABAergic neurons under control conditions, in the presence of picrotoxin $(1 \mu \mathrm{M})$, and in the presence of picrotoxin plus Ang-II. $\boldsymbol{C}, \mathrm{GABA}_{\mathrm{A}} \mathrm{R}$ blockade with picrotoxin attenuates Ang-IImediated increases in SNr GABAergic neuron APDs.

neurons. However, the activity of a single neuron is likely not to be representative of the population. Further, the combined input from numerous neurons encodes and sum to form the output characteristic of a neuronal population. Accordingly, to induce phasic synchronous neural activity in SNr GABAergic neurons, we used Thy1-ChR2YFP mice, which expresses ChR2 in SNr GABAergic neurons but not in SNc dopaminergic neurons (Arenkiel et al., 2007; Pan et al., 2013; Brown et al., 2014). To confirm whether Ang-II suppression of electrically-evoked firing of individual SNr GABAergic neurons occurs during phasic photoactivation of the population, we recorded EPSP (Fig. 8A) from SNr GABAergic neurons during photoactivation with a 100-ms-long 470-nm light pulse. Consistent with our earlier observation, Ang-II $(0.5 \mu \mathrm{M})$ decreased the number of intraburst spikes in four out of six recorded SNr GABAergic neurons obtained from six animals $\left(t_{(3)}=4.220,{ }^{*} p=0.024\right.$, paired Student's $t$ test; Fig. 8B,C). This observation is consistent with our other data showing negative modulatory effects of Ang-II on electrically evoked spike output of SNr GABAergic neurons.

SNr GABAergic neurons provide robust, monosynaptic inhibition of SNc dopaminergic neurons (Tepper et al., 1995; Tepper and Lee, 2007; Brazhnik et al., 2008; Pan et al., 2013). As an alternative approach to measure the effect of Ang-II on the output of SNr GABAergic neurons, we monitored the activity of postsynaptic dopaminergic neurons of the SNc by recording IPSC and IPSP from these cells during phasic (100 ms) photoactivation of $\mathrm{SNr}$ GABAergic neurons. In our other experiments, Ang-II suppressed electrical and light-evoked activity of $\mathrm{SNr}$ GABAergic neurons. Therefore, we expected that Ang-II would disinhibit postsynaptic SNc dopaminergic neurons because of decreased GABAergic input. Unexpectedly, we found that Ang-Il produced an $\sim 18 \%$ increase in the amplitude of light-evoked IPSC in SNc dopaminergic neurons $\left(n=10 ; t_{(9)}=2.270,{ }^{*} p=0.049\right.$; paired Student's $t$ test; Fig. 8F,G). Further, Ang-II also produced a proportional increase in the amplitude of light-evoked IPSP amplitude of these cells (Fig. 8E).

Together, these data indicate that Ang-II-mediated suppression of SNr GABAergic neurons, as shown in our preceding experiments, does not have a corresponding disinhibitory effect on postsynaptic SNc dopaminergic neurons during synchronous population activity. Rather, Ang-Il paradoxically strengthens feedforward inhibition of SNc dopaminergic neurons, suggesting a nonlinear effect of Ang-II on cellular activity and population output of $\mathrm{SNr}$ GABAergic neurons.

\section{Discussion}

In this study, we tested the hypothesis that Ang-II signaling in SN GABAergic neurons exerts an acute modulatory influence on mouse SNr GABAergic projection neurons and regulates inhibitory feedforward input to SNc dopaminergic neurons. 
A

control Ang-II
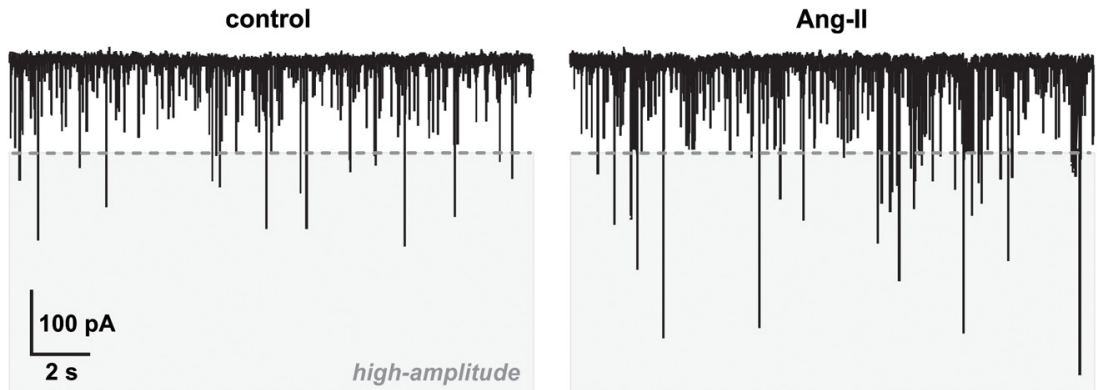

B
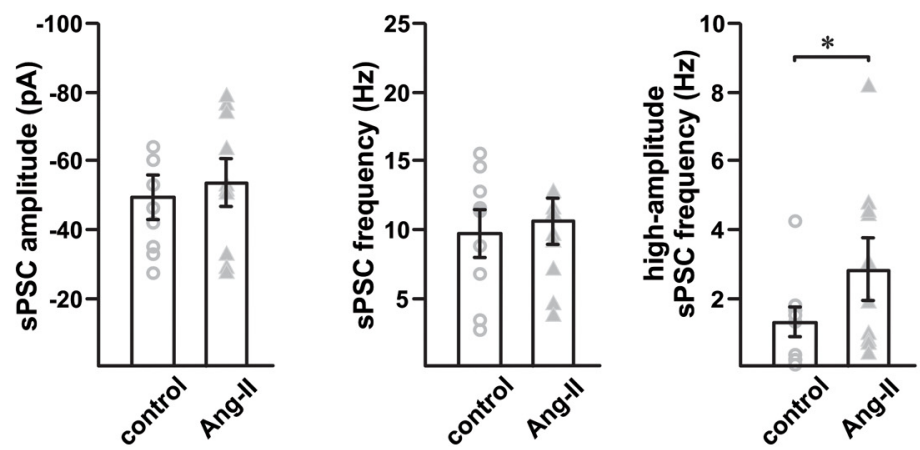

Figure 6. Ang-II increases SNr GABAergic neuron spontaneous PSCs. A, At a holding potential of $-65 \mathrm{mV}$ (junction potential corrected), spontaneous PSCs were recorded with a high chloride ( $57.5 \mathrm{~nm})$ internal pipette solution before and after 5 min of Ang-II (500 nM) exposure. $\boldsymbol{B}$, Ang-II did not markedly change the average PSC amplitude $(p=0.211$, paired Student's $t$ test) and frequency $(p=0.466$, paired Student's $t$ test) of spontaneous postsynaptic $\mathrm{Cl}^{-}$currents. However, Ang-Il significantly increased the observed incidence of high-amplitude spontaneous PSCs (right; ${ }^{*} p=0.012$; paired Student's $t$ test). High-amplitude spontaneous PSC, demarcated by the dashed line, were defined a priori as currents with an amplitude more than or equal to three times the mean current amplitude under control conditions.

\section{Canonical $\mathrm{AT}_{1}$ receptor signaling acutely and reversibly suppresses evoked APs in SNr GABAergic neurons}

In neurons identified as GABAergic, we found that acute application of Ang-II decreased evoked AP firing frequencies. We found that the effects of Ang-II on SNr GABAergic neurons were sensitive to $\mathrm{AT}_{1}$ receptor blockade by the specific antagonist losartan. This finding is consistent with $\mathrm{AT}_{1}$ receptor-mediated modulation of GABAergic neurotransmission in the amygdaloid complex and hypothalamus. Consistent with a dynamic regulatory mechanism, Ang-II suppression of SNr GABAergic AP firing was largely reversible on washout.

In contrast to the blockade of $\mathrm{AT}_{1}$ receptors with losartan, we observed no attenuation of Ang-II-dependent modulation of SNr GABAergic neuronal APs with the specific $A T_{2}$ receptor antagonist PD123319. Neither losartan nor PD123319 alone altered baseline AP firing characteristics in $\mathrm{SNr}$ GABAergic neurons. Additional angiotensin-related signaling modalities, such as other angiotensin molecules (e.g., IV and 1-7) and receptors (e.g., $\mathrm{AT}_{4}$ receptor and Mas receptors), could potentially participate in SNr GABAergic neuromodulation. Contributions by these mechanisms are likely minimal at best, given the relative abundance of Ang-II and $\mathrm{AT}_{1}$ receptors and our results with losartan blockade of $A T_{1}$ receptors.

\section{Ang-Il slows AP kinetics and increases firing variability of SNr GABAergic neurons}

Waveform analysis of Ang-II-responsive SNr GABAergic neuron APs revealed that Ang-II slows AP decay and rise, prolongs APD of evoked and spontaneous APs (except AP rise time), and also promoted variability in the pattern of $A P$ firing. These findings are consistent with reported changes in the electrical activity of several neurons exposed to Ang-II (Alioua et al., 2002; Zimmerman et al., 2005; Rainbow et al., 2009). Fast delayed rectifier Kv3.1 and Kv3.4 channels are primarily responsible for maintaining high sustained AP firing of SNr GABAergic neurons (Kaczmarek and Zhang, 2017; Ding et al., 2011; Zhou and Lee, 2011). Since AT 1 receptors reportedly inhibit delayed rectifier potassium channels in the hypothalamus and brain stem through a $\mathrm{G}_{\mathrm{q}}$-coupled, calcium/calmodulin-dependent protein kinase II-dependent pathway (Zhu et al., 1999); a similar mechanism may underlie the inhibitory effect of Ang-II on SNr GABAergic neurons. Future investigations are necessary to identify and mechanistically characterize ion channels potentially modulated by $\mathrm{AT}_{1}$ receptors in these cells.

\section{Ang-II activates postsynaptic GABA $A_{A} R$ in $\mathrm{SNr}$ GABAergic neurons}

SNr GABAergic neurons receive robust inhibitory input from the striatum and external globus pallidus (Smith and Bolam, 1990; Lee et al., 2004; Deniau et al., 2007). Postsynaptic $\mathrm{GABA}_{\mathrm{A}}$ Rs are the dominant inhibitory mechanism of these neurons (Precht and Yoshida, 1971; Boyes and Bolam, 2007). Our data show that $\mathrm{GABA}_{\mathrm{A}} \mathrm{R}$ blockade with picrotoxin prevents Ang-II-mediated suppression of evoked firing in SNr GABAergic neurons. We also observed an $\sim 2.5$-fold increase in picrotoxin-sensitive high- 
A

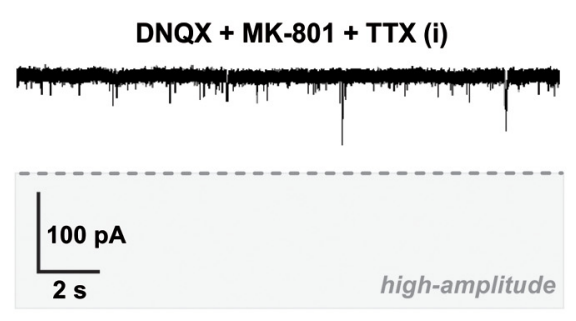

B

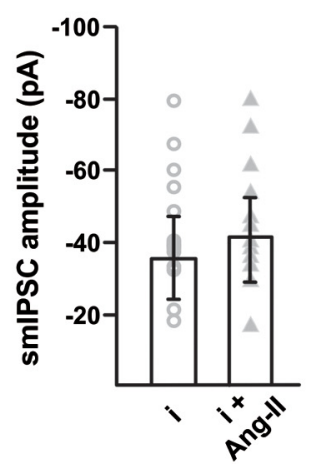

GABAergic
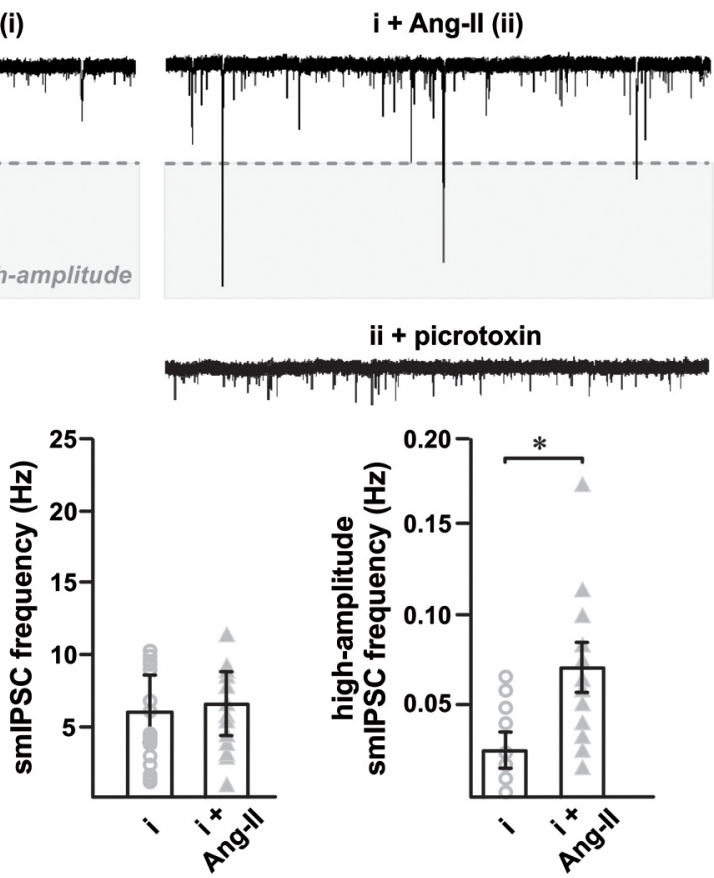

ii + picrotoxin

C

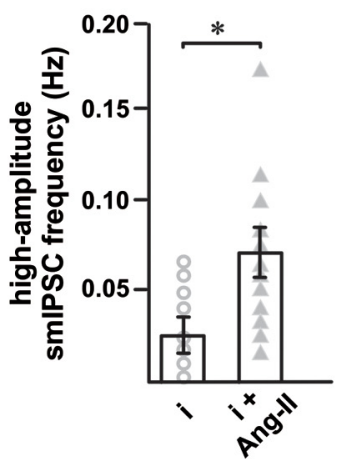

dopaminergic

DNQX + MK-801 (i)
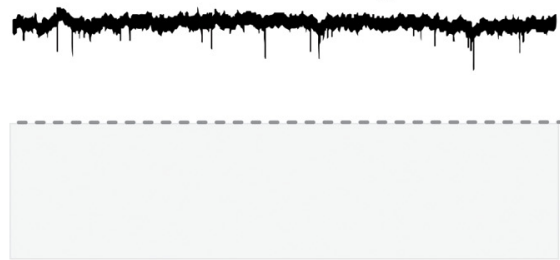

i + TTX (ii)

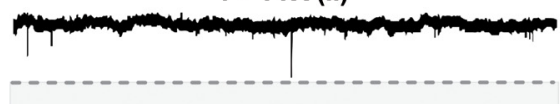

Figure 7. Ang-II increases picrotoxin-sensitive spontaneous MIPSC in SNr GABAergic neurons. Spontaneous mIPSC recorded from SNr GABAergic and SNc dopaminergic neurons in the presence of excitatory synaptic blockers (DNQX, MK-801; $1 \mu \mathrm{M})$ and TTX (500 nм). A, Representative traces showing Ang-II-mediated enhancement of high-amplitude postsynaptic GABA A $_{A}$ currents. Highamplitude spontaneous PSCs, demarcated by the dashed line, were defined a priori as currents with an amplitude more than or equal to three times the mean current amplitude recorded in the presence of excitatory synaptic blockers and TTX. B, Summary data showing Ang-II-mediated $\sim 2.5$-fold increase in high-amplitude mIPSC ( ${ }^{*} p=0.019$, one-way repeated measures ANOVA), but not in average mIPSC amplitude and frequency. C, In contrast to SNr GABAergic neurons, Ang-II had no detectable effect on spontaneous mIPSC in SNc dopaminergic neurons.

amplitude mIPSC with Ang-II, but no noticeable change in average frequency and amplitude. This observation suggests that Ang-Il activates postsynaptic GABA $A_{A} R$ s in SN GABAergic neurons. Consistent with this hypothesis, Ang-II-mediated facilitation of $\mathrm{GABA}_{A} \mathrm{R}$ activity reportedly occurs in neurons of the rat median preoptic nucleus (Henry et al., 2009).

We also recorded SNr GABAergic mIPSC following local Ang-II application; however, we observed no change in mIPSC properties. However, subsequent bath perfusion of Ang-Il did increase the occurrence of high-amplitude mIPSC. This observation suggests that $\mathrm{GABA}_{A} \mathrm{Rs}$ potentiated by Ang-II are most likely expressed in distal dendritic terminals away from the soma and embedded deep in the slice. Further investigation is necessary to determine the location and mechanism underlying the coupling between $A T_{1}$ receptors and $G A B A_{A} R s$ in $S N r$ GABAergic neurons.

\section{Ang-Il does not change the AP kinetics and basal IPSC in SNc dopaminergic neurons}

Evidence suggests that Ang-II via $A T_{1}$ receptors impacts SNc dopaminergic neuronal function, homeostasis, and viability (Grammatopoulos et al., 2007; Garrido-Gil et al., 2013; Sonsalla et al., 2013; Labandeira-García et al.,
2014). At present, it is unclear whether the effects of AngII on SNc dopaminergic neurons are direct, indirect, or a combination of both. To address this issue, we recorded evoked AP, PSC, and IPSC from SNc dopaminergic neurons. We could not reliably obtain interpretable AP firing frequency data because of the well-characterized adaptive response of dopaminergic neurons to depolarizing currents (data not shown). However, unlike SNr GABAergic neurons, we found that Ang-Il had no apparent effect on the AP kinetics, PSC, and IPSC of SNc dopaminergic neurons, suggesting a heterogeneous effect in the two types of SN neurons.

The apparent lack of effect by Ang-II on SN dopaminergic neurons in our experiments could result from a low expression of $\mathrm{GABA}_{A} \mathrm{Rs}$, different subunit compositions, or a lack of canonical $\mathrm{KCC} 2 \mathrm{~K}^{+}-\mathrm{Cl}^{-}$co-transporters (Boyes and Bolam, 2007; Paladini and Tepper, 2016). SNr GABAergic projection neurons express KCC2 co-transporters, which actively pump $\left[\mathrm{Cl}^{-}\right]$out of the cell to maintain a hyperpolarized (around $-71 \mathrm{mV}$ ) chloride reversal potential (Rivera et al., 1999). In contrast, the chloride reversal potential is around $-63 \mathrm{mV}$ in SNc dopaminergic neurons because of a lack of KCC2 expression (Gulácsi et al., 2003). In this scenario, $G_{A B A} R$ potentiation could produce a greater hyperpolarization in SNr GABAergic neurons than SNc dopaminergic neurons. 
A

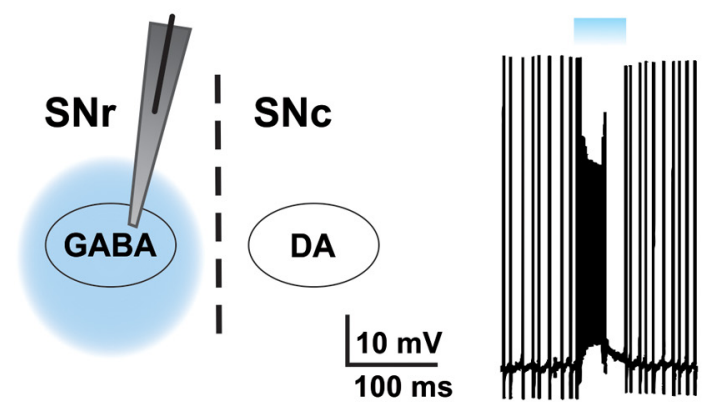

D

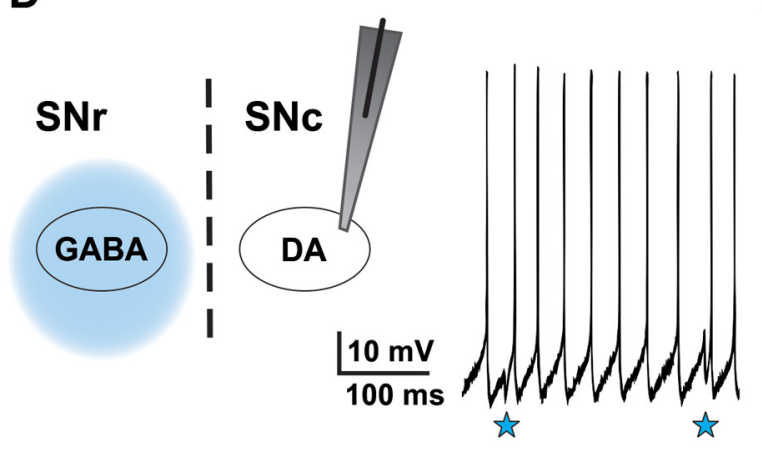

B

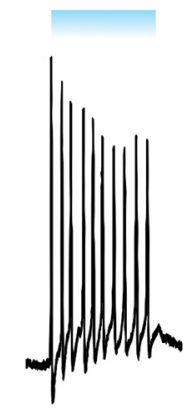

control

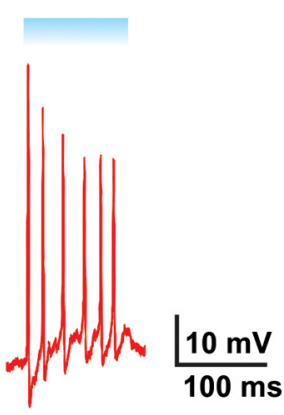

Ang-II
C

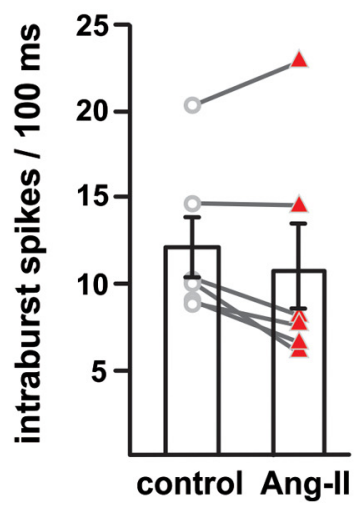

E

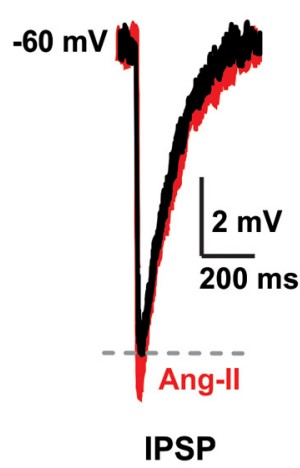

$\mathbf{F}$

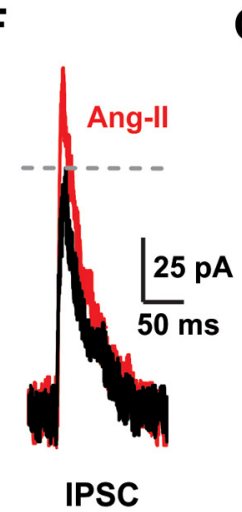

G

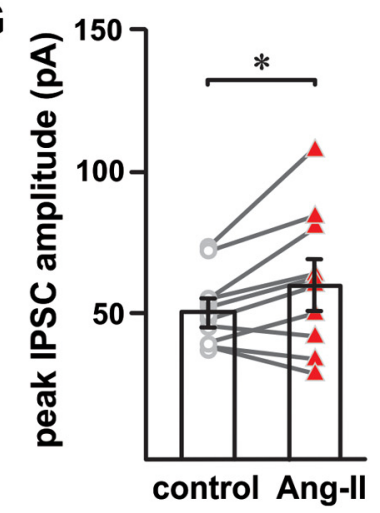

Figure 8. Ang-II modulates SN GABAergic neurotransmission. A, Light-evoked EPSP (bursts) in SNr GABAergic neurons in response to a 100-ms-long light pulse. $\boldsymbol{B}$, Ang-ll decreased light-evoked intraburst spikes in four out of six SNr GABAergic neurons $\left({ }^{*} p=0.024\right.$, paired Student's $t$ test); intraburst spikes shown are enlarged from panel $\boldsymbol{A}$. $\boldsymbol{C}$, Summary data showing the effect of Ang-II on intraburst spikes (EPSP) in SNr GABAergic neurons in response to 100-ms-long light pulse. In four out of six SNr GABAergic neurons, Ang-Il decreased intraburst spikes by $\sim 25 \%$. $\boldsymbol{D}$, IPSP recorded from SNc dopaminergic neurons in response to photostimulation of SNr GABAergic neurons, as shown by downward deflection of membrane potential (blue stars). Ang-II enhanced light-evoked inhibitory input onto SNc dopaminergic neurons as shown by an increase in the amplitude of light-evoked IPSP $(\boldsymbol{E})$ and IPSC $\left(\boldsymbol{F}, \mathbf{G} ;{ }^{*} p=0.049\right.$, paired Student's $t$ test).

\section{Ang-II decreases light-evoked EPSP in SNr GABAergic neurons}

During periods of synchronous activity, localized feedback inhibition within the SNr limits SNr GABAergic neuronal output (Brown et al., 2014). This inhibitory mechanism involves SNr GABAergic axon collaterals, which synapse not only with dopaminergic neurons in the SNc but also with SNc GABAergic neurons. The resultant collateral inhibition provides a robust gain control of the total GABAergic neuronal output (Tepper et al., 1995; Tepper and Lee, 2007; Brown et al., 2014).

Using transgenic mice with GABAergic neuron-specific expression of ChR2 (THY1-ChR2-YFP), we examined the negative modulatory effect of Ang-II on SNr GABAergic neurons during synchronous phasic activity. Consistent with our electrically-evoked experiments, we found that Ang-II decreased light-evoked EPSP (intraburst suprathreshold spikes) in four out of six SNr GABAergic neurons. These data further confirm the inhibitory nature of Ang-II on SNr GABAergic neurons. Given the complex and non-uniform arborizations of the SNr GABAergic axon collaterals, we suggest that the effect of Ang-II on $\mathrm{SNr}$ GABAergic neurons is likely to be heterogeneous.
The response of an individual SNr GABAergic neuron to Ang-Il during phasic stimulation will depend on the extent of the parallel connections with neighboring GABAergic neurons. As such, the effect of Ang-II on SNr GABAergic output is complicated further by the influence of heterogenous intranigral feedback activity.

\section{Ang-II enhances feedforward inhibitory input to SNc dopaminergic neurons}

SNr GABAergic neurons provide robust, monosynaptic inhibition of SNc dopaminergic neurons (Tepper et al., 1995; Tepper and Lee, 2007; Brazhnik et al., 2008; Pan et al., 2013). Ang-II-mediated suppression of SNr GABAergic output should, therefore, have a disinhibitory effect on SNc dopaminergic neurons. We observed, unexpectedly, that Ang-II increased the amplitude of SNc dopaminergic neuron IPSC and IPSP in response to photostimulation of SNr GABAergic neurons. The paradoxical strengthening of feedforward input from SNr GABAergic neurons is inconsistent with our other data showing Ang-II-mediated suppression of SNr GABAergic neurons. This nonlinear effect of 
Ang-II on SNr GABAergic neurons and their output to postsynaptic SNc dopaminergic neurons suggests a complex mechanism originating either from synaptic changes induced by Ang-II and the nature of intranigral circuitry. Subsequent investigations are needed to explore these intriguiging effects of Ang-II.

SNr GABAergic projection neurons have extensive intranigral and extranigral collateralizations. Non-uniformity of these branchings can further contribute to variations in excitability, synaptic bouton content, and ion channel distributions within the network (Debanne et al., 2011; Martin et al., 2014; Seidl, 2014; Bucher, 2016). In such a complex circuit arising from a heterogeneous and interlinked network, the summed activity could result in a range of synchronous or asynchronous population activities. Therefore, the paradoxical facilitation of inhibitory input to SNc dopaminergic neurons by AngII on phasic activation of SNr GABAergic neurons could be explained, at least in part, by the complex and asymmetrical nature of the intranigral microcircuitry.

The mechanisms by which $A T_{1}$ receptors mediate the Ang-II-dependent modulation of GABAergic neuronal activity are unclear. $A T_{1}$ receptors generally couple with $G_{q^{-}}$ proteins and initiate multiple intracellular signaling cascades (Seshiah et al., 2002; Mehta and Griendling, 2007). Canonical $\mathrm{AT}_{1}$ receptor signaling often involves phospholipase C-dependent generation of diacylglycerol and inositol triphosphate, which activates numerous target proteins and associated signaling cascades. Major downstream $\mathrm{AT}_{1}$ receptor targets include PKC, MAP kinases, receptor and nonreceptor tyrosine kinases, and scaffold proteins. Further, $A T_{1}$ receptor activation leads to reactive oxygen species (ROS) generation via NADPH oxidase and increases the synthesis of endocannabinoids (Melis and Pistis, 2007; Choi et al., 2012; Szekeres et al., 2012; Gyires et al., 2014; Labandeira-García et al., 2014). Future investigations are necessary to delineate and characterize the specific mechanisms that modulate GABAergic and dopaminergic neuronal activity in the substantia nigra.

From these data, we conclude that Ang-Il signaling occurs in the SNr GABAergic neurons via a postsynaptic $A T_{1}$ receptor-dependent mechanism. Our data show enhancement of postsynaptic GABA $A_{A}$ Rs and a slowing of AP kinetics by Ang-II as contributing factors for the suppressive effect of Ang-II in SNr GABAergic neurons. However, the inhibitory effect of Ang-II on SNr GABAergic neurons did not translate to disinhibition of postsynaptic SNc dopaminergic neurons. Rather, we observed a paradoxical increase in postsynaptic inhibitory input. This observation implies a nonlinear effect of Ang-II on GABAergic neurotransmission on postsynaptic SNc dopaminergic neurons. Further investigation into the microcircuit dynamics, underlying signaling cascades and effector proteins (e.g., ion channels), and the ensuing effects on SNc dopaminergic neurons by Ang-II, is warranted.

\section{References}

Alioua A, Mahajan A, Nishimaru K, Zarei MM, Stefani E, Toro L (2002) Coupling of c-Src to large conductance voltage- and $\mathrm{Ca}^{2+}$-activated $\mathrm{K}^{+}$channels as a new mechanism of agonist-induced vasoconstriction. Proc Natl Acad Sci USA 99:14560-14565.

Allen AM, Paxinos G, McKinley MJ, Chai SY, Mendelsohn FA (1991) Localization and characterization of angiotensin II receptor binding sites in the human basal ganglia, thalamus, midbrain pons, and cerebellum. J Comp Neurol 312:291-298.

Arenkiel BR, Peca J, Davison IG, Feliciano C, Deisseroth K, Augustine GJ, Ehlers MD, Feng G (2007) In vivo light-induced activation of neural circuitry in transgenic mice expressing channelrhodopsin-2. Neuron 54:205-218.

Arnold AC, Gallagher PE, Diz DI (2013) Brain renin-angiotensin system in the nexus of hypertension and aging. Hypertens Res 36:5-13.

Atherton JF, Bevan MD (2005) lonic mechanisms underlying autonomous action potential generation in the somata and dendrites of GABAergic substantia nigra pars reticulata neurons in vitro. $J$ Neurosci 25:8272-8281.

Barry PH (1994) JPCalc, a software package for calculating liquid junction potential corrections in patch-clamp, intracellular, epithelial and bilayer measurements and for correcting junction potential measurements. J Neurosci Methods 51:107-116.

Boyes J, Bolam JP (2007) Localization of GABA receptors in the basal ganglia. Prog Brain Res 160:229-243.

Brazhnik E, Shah F, Tepper JM (2008) GABAergic afferents activate both $\mathrm{GABA}_{A}$ and $\mathrm{GABA}_{B}$ receptors in mouse substantia nigra dopaminergic neurons in vivo. J Neurosci 28:10386-10398.

Brown DC, Steward LJ, Ge J, Barnes NM (1996) Ability of angioten$\sin$ II to modulate striatal dopamine release via the $A T_{1}$ receptor in vitro and in vivo. Br J Pharmacol 118:414-420.

Brown J, Pan WX, Dudman JT (2014) The inhibitory microcircuit of the substantia nigra provides feedback gain control of the basal ganglia output. Elife 3:e02397.

Bucher D (2016) Contribution of Axons to Short-Term Dynamics of Neuronal Communication. In, Axons and Brain ArchitectureAxons and Brain Architecture, pp 245-263. Boston, MA:Elsevier.

Choi DH, Cristóvão AC, Guhathakurta S, Lee J, Joh TH, Beal MF, Kim YS (2012) NADPH oxidase 1 mediated oxidative stress leads to dopamine neuron death in Parkinson's disease. Antioxid Redox Signal 16:1033-1045.

Debanne D, Campanac E, Bialowas A, Carlier E, Alcaraz G (2011) Axon physiology. Physiol Rev 91:555-602.

Deniau JM, Mailly P, Maurice N, Charpier S (2007) The pars reticulata of the substantia nigra: a window to basal ganglia output. Prog Brain Res 160:151-172.

Ding S, Matta SG, Zhou FM (2011) Kv3-like potassium channels are required for sustained high-frequency firing in basal ganglia output neurons. J Neurophysiol 105:554-570.

Dray A (1979) The striatum and substantia nigra: a commentary on their relationships. Neuroscience 4:1407-1439.

Garrido-Gil P, Valenzuela R, Villar-Cheda B, Lanciego JL, Labandeira-García JL (2013) Expression of angiotensinogen and receptors for angiotensin and prorenin in the monkey and human substantia nigra: an intracellular renin-angiotensin system in the nigra. Brain Struct Funct 218:373-388.

Ge J, Barnes NM (1996) Alterations in angiotensin $\mathrm{AT}_{1}$ and $\mathrm{AT}_{2}$ receptor subtype levels in brain regions from patients with neurodegenerative disorders. Eur J Pharmacol 297:299-306.

Grammatopoulos TN, Jones SM, Ahmadi FA, Hoover BR, Snell LD, Skoch J, Jhaveri VV, Poczobutt AM, Weyhenmeyer JA, Zawada WM (2007) Angiotensin type 1 receptor antagonist losartan, reduces MPTP-induced degeneration of dopaminergic neurons in substantia nigra. Mol Neurodegener 2:1.

Gulácsi A, Lee CR, Sík A, Viitanen T, Kaila K, Tepper JM, Freund TF (2003) Cell type-specific differences in chloride-regulatory mechanisms and $\mathrm{GABA}(\mathrm{A})$ receptor-mediated inhibition in rat substantia nigra. J Neurosci 23:8237-8246.

Gyires K, Rónai AZ, Zádori ZS, Tóth VE, Németh J, Szekeres M, Hunyady L (2014) Angiotensin II-induced activation of central AT receptors exerts endocannabinoid-mediated gastroprotective effect in rats. Mol Cell Endocrinol 382:971-978.

Henry M, Grob M, Mouginot D (2009) Endogenous angiotensin II facilitates GABAergic neurotransmission afferent to the $\mathrm{Na}^{+}$-responsive neurons of the rat median preoptic nucleus. Am J Physiol Regul Integr Comp Physiol 297:R783-R792. 
Hikosaka O (2007) GABAergic output of the basal ganglia. Prog Brain Res 160:209-226.

Hu B, Qiao H, Cao T, Sun B, Luo X, Jia R, Fan Y, Wang N, Lu Y, Yan $\mathrm{J}$ (2018) Angiotensin II facilitates GABAergic neurotransmission at postsynaptic sites in rat amygdala neurons. Neuropharmacology 133:334-344.

Jackson L, Eldahshan W, Fagan SC, Ergul A (2018) Within the brain: the renin angiotensin system. Int J Mol Sci 19:876.

Kaczmarek LK, Zhang Y (2017) Kv3 channels: enablers of rapid firing, neurotransmitter release, and neuronal endurance. Physiol Rev 97:1431-1468.

Labandeira-García JL, Garrido-Gil P, Rodriguez-Pallares J, Valenzuela R, Borrajo A, Rodriguez-Perez Al (2014) Brain renin-angiotensin system and dopaminergic cell vulnerability. Front Neuroanat 8:67.

Labandeira-García JL, Rodriguez-Perez Al, Garrido-Gil P, RodriguezPallares J, Lanciego JL, Guerra MJ (2017) Brain renin-angiotensin system and microglial polarization: implications for aging and neurodegeneration. Front Aging Neurosci 9:129.

Lacey M, Mercuri N, North R (1989) Two cell types in rat substantia nigra zona compacta distinguished by membrane properties and the actions of dopamine and opioids. J Neurosci 9:1233-1241.

Lee CR, Abercrombie ED, Tepper JM (2004) Pallidal control of substantia nigra dopaminergic neuron firing pattern and its relation to extracellular neostriatal dopamine levels. Neuroscience 129:481489.

Lobb CJ, Wilson CJ, Paladini CA (2011) High-frequency, short-latency disinhibition bursting of midbrain dopaminergic neurons. $J$ Neurophysiol 105:2501-2511.

Mailly P, Charpier S, Menetrey A, Deniau JM (2003) Three-dimensional organization of the recurrent axon collateral network of the substantia nigra pars reticulata neurons in the rat. $\mathrm{J}$ Neurosci 23:5247-5257.

Martin KAC, Roth S, Rusch ES (2014) Superficial layer pyramidal cells communicate heterogeneously between multiple functional domains of cat primary visual cortex. Nat Commun 5:5252.

McGeer EG, Staines WA, McGeer PL (1984) Neurotransmitters in the basal ganglia. Can J Neurol Sci 11:89-99.

Mehta PK, Griendling KK (2007) Angiotensin II cell signaling: physiological and pathological effects in the cardiovascular system. Am J Physiol Cell Physiol 292:C82-C97.

Melis M, Pistis M (2007) Endocannabinoid signaling in midbrain dopamine neurons: more than physiology? Curr Neuropharmacol 5:268-277.

Paladini CA, Tepper JM (2016) Chapter 17 - neurophysiology of substantia nigra dopamine neurons: modulation by GABA and glutamate. In: Handbook of behavioral neuroscience, pp 335-360. Amsterdam: Elsevier.

Pan WX, Brown J, Dudman JT (2013) Neural signals of extinction in the inhibitory microcircuit of the ventral midbrain. Nat Neurosci 16:71-78.

Parent A (1990) Extrinsic connections of the basal ganglia. Trends Neurosci 13:254-258.

Perez-Lloret S, Otero-Losada M, Toblli JE, Capani F (2017) Reninangiotensin system as a potential target for new therapeutic approaches in Parkinson's disease. Expert Opin Investig Drugs 26:1163-1173.

Precht W, Yoshida M (1971) Blockage of caudate-evoked inhibition of neurons in the substantia nigra by picrotoxin. Brain Res 32:229233.

Rainbow RD, Norman RI, Everitt DE, Brignell JL, Davies NW, Standen NB (2009) Endothelin-I and angiotensin II inhibit arterial voltage-gated $\mathrm{K}^{+}$channels through different protein kinase $\mathrm{C}$ isoenzymes. Cardiovasc Res 83:493-500.

Rice ME, Patel JC, Cragg SJ (2011) Dopamine release in the basal ganglia. Neuroscience 198:112-137.
Richards CD, Shiroyama T, Kitai ST (1997) Electrophysiological and immunocytochemical characterization of GABA and dopamine neurons in the substantia nigra of the rat. Neuroscience 80:545557.

Rivera C, Voipio J, Payne JA, Ruusuvuori E, Lahtinen H, Lamsa K, Pirvola U, Saarma M, Kaila K (1999) The $\mathrm{K}^{+} / \mathrm{Cl}^{-}$co-transporter $\mathrm{KCC} 2$ renders GABA hyperpolarizing during neuronal maturation. Nature 397:251-255.

Rocha NP, Scalzo PL, Barbosa IG, de Campos-Carli SM, Tavares LD, de Souza MS, Christo PP, Reis HJ, Simões E Silva AC, Teixeira AL (2016) Peripheral levels of angiotensins are associated with depressive symptoms in Parkinson's disease. J Neurol Sci 368:235-239.

Seidl AH (2014) Regulation of conduction time along axons. Neuroscience 276:126-134.

Seshiah PN, Weber DS, Rocic P, Valppu L, Taniyama Y, Griendling KK (2002) Angiotensin II stimulation of NAD(P)H oxidase activity: upstream mediators. Circ Res 91:406-413.

Seutin V, Engel D (2010) Differences in $\mathrm{Na}^{+}$conductance density and $\mathrm{Na}^{+}$channel functional properties between dopamine and GABA neurons of the rat substantia nigra. J Neurophysiol 103:3099-3114.

Simonnet G, Giorguieff-Chesselet MF (1979) Stimulating effect of angiotensin II on the spontaneous release of newly synthetized $\left[{ }^{3} \mathrm{H}\right]$ dopamine in rat striatal slices. Neurosci Lett 15:153-158.

Smith Y, Bolam JP (1990) The output neurones and the dopaminergic neurones of the substantia nigra receive a GABA-containing input from the globus pallidus in the rat. J Comp Neurol 296:47-64.

Sonsalla PK, Coleman C, Wong LY, Harris SL, Richardson JR, Gadad BS, Li W, German DC (2013) The angiotensin converting enzyme inhibitor captopril protects nigrostriatal dopamine neurons in animal models of parkinsonism. Exp Neurol 250:376-383.

Szekeres M, Nádasy GL, Turu G, Soltész-Katona E, Tóth ZE, Balla A, Catt KJ, Hunyady L (2012) Angiotensin II induces vascular endocannabinoid release, which attenuates its vasoconstrictor effect via CB1 cannabinoid receptors. J Biol Chem 287:31540-31550.

Tepper JM, Lee CR (2007) GABAergic control of substantia nigra dopaminergic neurons. Prog Brain Res 160:189-208.

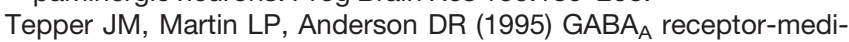
ated inhibition of rat substantia nigra dopaminergic neurons by pars reticulata projection neurons. J Neurosci 15:3092-3103.

Valenzuela R, Costa-Besada MA, Iglesias-Gonzalez J, Perez-Costas E, Villar-Cheda B, Garrido-Gil P, Melendez-Ferro M, Soto-Otero R, Lanciego JL, Henrion D, Franco R, Labandeira-García JL (2016) Mitochondrial angiotensin receptors in dopaminergic neurons. Role in cell protection and aging-related vulnerability to neurodegeneration. Cell Death Dis 7:e2427.

Xing J, Lu J, Li J (2009) Angiotensin II inhibits GABAergic synaptic transmission in dorsolateral periaqueductal gray neurons. Neurosci Lett 455:8-13.

Yung WH, Häusser MA, Jack JJ (1991) Electrophysiology of dopaminergic and non-dopaminergic neurones of the guinea-pig substantia nigra pars compacta in vitro. J Physiol 436:643-667.

Zhou FM, Lee CR (2011) Intrinsic and integrative properties of substantia nigra pars reticulata neurons. Neuroscience 198:69-94.

Zhou FW, Matta SG, Zhou FM (2008) Constitutively active TRPC3 channels regulate basal ganglia output neurons. J Neurosci 28:473-482.

Zhu M, Gelband CH, Posner P, Sumners C (1999) Angiotensin II decreases neuronal delayed rectifier potassium current: role of calcium/calmodulin-dependent protein kinase II. J Neurophysiol 82:1560-1568.

Zimmerman MC, Sharma RV, Davisson RL (2005) Superoxide mediates angiotensin II-induced influx of extracellular calcium in neural cells. Hypertension 45:717-723. 